\title{
Évolution chimique des sols en exploitations d'élevage intensif : exemple du Finistère
}

\author{
M Coppenet ${ }^{1}$, J Golven 2, JC Simon ${ }^{1 *}$, L Le Corre 1, M Le Roy ${ }^{1}$ \\ 1 INRA, Station d'agronomie, 4, rue Stang-Vihan; \\ ${ }^{2}$ Chambre d'Agriculture, 5, allée Sully, 29000 Quimper, France \\ MINISTERE de L'AGRICULTURE \\ STATION de GENETLQUUE \\ et d'AMELIOAATION des PLATiUIES \\ INRA B.V. 1540 \\ 21034 DIJON CEDEX \\ (Reçu le 15 mai 1992; accepté le 4 décembre 1992) \\ FRANCE
}

\begin{abstract}
Résumé - Les exploitations d'élevage de Bretagne (porcs, laitières, volailles) se caractérisent par leur caractère intensif. Le calcul théorique des bilans minéraux à l'échelle de l'exploitation, effectué dès 1973, mettait en évidence des excès importants pour $\mathrm{P}, \mathrm{K}$, Cu et $\mathrm{Zn}$. Ces éléments sont issus en grande partie des lisiers et des fumiers. Afin de vérifier expérimentalement les conséquences de ces excès sur la teneur du sol, la station d'agronomie de Quimper a mis en place un réseau d'observation dans 64 fermes d'élevage. Les modifications chimiques du sol sont suivies tous les 3 ans, sur 190 parcelles du Finistère. Après 15 années, un premier bilan peut être dressé. On observe, comme prévu, un très fort enrichissement des sols en $\mathrm{P}_{2} \mathrm{O}_{5}$ (Dyer-Demolon), Cu et $\mathrm{Zn}$ (EDTA), les augmentations moyennes de teneur étant respectivement de 28,0,22 et 0,37 ppm par an. L'enrichissement mis en évidence pour le potassium est d'autant plus important que la teneur initiale du sol est faible, l'ensemble des teneurs tendant vers une limite supérieure voisine de $400 \mathrm{mg} \mathrm{K}$ O/ $\mathrm{kg}$. En raison de l'additivité de la phytotoxicité du cuivre et du zinc, des problèmes risquent d'apparaître dans les exploitations les plus intensives à échéance d'un siècle.
\end{abstract}

lisier / phosphore / potassium / cuivre / zinc

\begin{abstract}
Summary - Chemical evolution of soils in intensive animal-rearing farms: the example of Finistère. In Brittany (western France), pig, cattle and poultry breeding are particularly intensive. A theoretical nutrient balance at the farm level shows important excesses for phosphorus, potassium, copper and zinc. These excesses could have an incidence on chemical evolution of soils due to the addition of slurry. To study this potential soil enrichment, the Quimper Agronomic Station started a survey in 1973 at 64 breeding farms. One hundred and ninety soils were analysed every $3 \mathrm{yr}$. This survey showed a soil enrichment in $\mathrm{P}_{2} \mathrm{O}_{5}, \mathrm{Cu}$ and $\mathrm{Zn}$ : the average increases were 28, 0.22 and $0.37 \mathrm{ppm}$ respectively per yr. Potassium content is also increasing in relation to initial soil content, and approached an upper limit close to $400 \mathrm{mg} \mathrm{K} \mathrm{K}_{2} \mathrm{O} / \mathrm{kg}$. As copper and zinc toxicities appear to be additive, phytotoxicity could occur after only 1 century in the most intensive breeding farms.
\end{abstract}

slurry / phosphorus / potassium / copper / zinc

\section{INTRODUCTION}

Au début des années 70 , la station d'agronomie de Quimper a engagé une réflexion sur les bilans des éléments minéraux à l'échelle de l'exploitation, en région d'élevage intensif (Coppenet, 1972, 1974 et 1975). Ces bilans théoriques mettaient en évidence des excédents importants pour les éléments minéraux majeurs $(N, P, K)$, ainsi que pour deux oligo-éléments en élevage porcin (Cu et $\mathrm{Zn}$ ).
Le calcul d'un tel bilan (Coppenet, 1974; Coppenet, 1985) repose sur une estimation des entrées (par les engrais minéraux et les aliments du bétail achetés) et des sorties (par les produits animaux et végétaux vendus et les pertes par lessivage).

Dans le cas d'une exploitation porcine où les animaux consomment le maïs grain produit sur place, l'éleveur peut engraisser 35 porcs charcutiers de $100 \mathrm{~kg} \mathrm{ha}^{-1} . \mathrm{an}^{-1}$, sous réserve d'apporter en complément 23 quintaux de soja et 2,5

\footnotetext{
* Correspondance et tirés à part
} 
quintaux de complément minéral. Le calcul met en évidence un net excédent pour les 4 éléments considérés (Coppenet, 1974) : + $59 \mathrm{~kg}$ $\mathrm{P}_{2} \mathrm{O}_{5} / \mathrm{ha} / \mathrm{an},+31 \mathrm{~kg} \mathrm{~K}{ }_{2} \mathrm{O} / \mathrm{ha} / \mathrm{an},+1,32 \mathrm{Cu} / \mathrm{ha} / \mathrm{an}$ et $+1,37 \mathrm{~kg} \mathrm{Zn/ha/an}$.

De tels bilans théoriques, établis pour d'autres types de productions animales, montrent également des excédents importants :

- porcs hors-sol : tous les aliments sont achetés, et pour une production animale égale à celle de l'exemple ci-dessus, l'excès est de $200 \mathrm{~kg} \mathrm{P}_{2} \mathrm{O}_{5}$ $\mathrm{ha}^{-1} \cdot \mathrm{an}^{-1}$ et de $150 \mathrm{~kg} \mathrm{~K}_{2} \mathrm{O} \mathrm{ha}^{-1} \cdot \mathrm{an}^{-1}$;

- élevage de taurillons consommant l'ensilage de maïs produit sur l'exploitation : un calcul analogue conduit à un excédent de $39 \mathrm{~kg} \mathrm{P}_{2} \mathrm{O}_{5}$ ha ${ }^{-1} \cdot$ an $^{-1}$ et $27 \mathrm{~kg} \mathrm{~K}_{2} \mathrm{O}$ ha $^{-1}$. an-1;

- élevage laitier produisant 10000 I de lait/ha/ an, avec un assolement à base de maïs et de ray-grass : le calcul conduit à un bilan nul ou légèrement positif en $\mathrm{P}_{2} \mathrm{O}_{5}$ et à un déficit en potasse de $25 \mathrm{~kg} \cdot \mathrm{ha}^{-1} \cdot \mathrm{an}^{-1}$.

On parvient ainsi à des bilans généralement positifs pour les grands types d'exploitations agricoles d'élevage intensif. Ces excès d'élements minéraux, dont une grande partie se retrouve dans les déjections animales, retournent au sol sous forme de lisiers ou fumiers, ou de bouses et pissats dans le cas de restitutions directes au pâturage. Pour les éléments qui lessivent peu $(\mathrm{P}, \mathrm{Cu}$ et $\mathrm{Zn})$ ou modérément $(\mathrm{K})$, on pouvait prévoir dès le début des années 70 , un enrichissement progressif des sols (Coppenet, 1974).

Afin de vérifier expérimentalement cette hypothèse, la station d'agronomie de Quimper, en étroite collaboration avec la Chambre d'agriculture du Finistère, a constitué dès 1973 un réseau d'observation en exploitations d'élevage intensif, afin de suivre les modifications chimiques des sols recevant de fortes doses de lisier.

Cet article présente les évolutions de teneurs observées dans les sols au cours de 15 années de suivi et de 6 campagnes de mesures.

\section{MATÉRIEL ET MÉTHODES}

L'objectif était de mettre en évidence les modifications chimiques éventuelles de la couche arable des sols d'un nombre représentatif d'exploitations agricoles en élevage intensif. Pour y répondre, on a procédé tous les 3 ans, à partir de 1973, à des analyses de sols issus de 64 exploitations du Finistère. Ces dernières recouvrent assez bien la diversité pédoclimatique du département : des zones côtières aux températures douces et aux précipitations modérées (800 à 900 $\mathrm{mm}$ ) aux régions intérieures plus froides et très humides (1 200 à $1500 \mathrm{~mm}$ ); des sols granitiques riches en matière organique ( $6 \%$ en moyenne) aux sols sur schistes en passant par les sols de limon pour les exploitations situées dans la zone légumière au nord (fig 1).

\section{Systèmes de production animale}

Au début de l'enquête, la répartition des exploitations en fonction du type de production animale était telle que présentée dans le tableau I.

En 1973, les exploitations choisies étaient à dominance porcine (50 sur 64 élèvent des porcs). Depuis, on a noté une forte progression des exploitations spécialisées dans cette seule production (27 contre 19) au détriment de la production laitière (incidence des quotas laitiers). Les densités porcines de ces élevages sont en moyenne de 40 porcs logés/ha de SAU, ce qui correspond à une production annuelle de 100 porcs charcutiers/ha. Cinq élevages dépassent cependant les 100 porcs logés/ha. Les densités porcines sont plus faibles dans les élevages avec vaches laitières : en moyenne, 30 porcs logés/ha de SAU, soit 75 porcs charcutiers produits/ha/an.

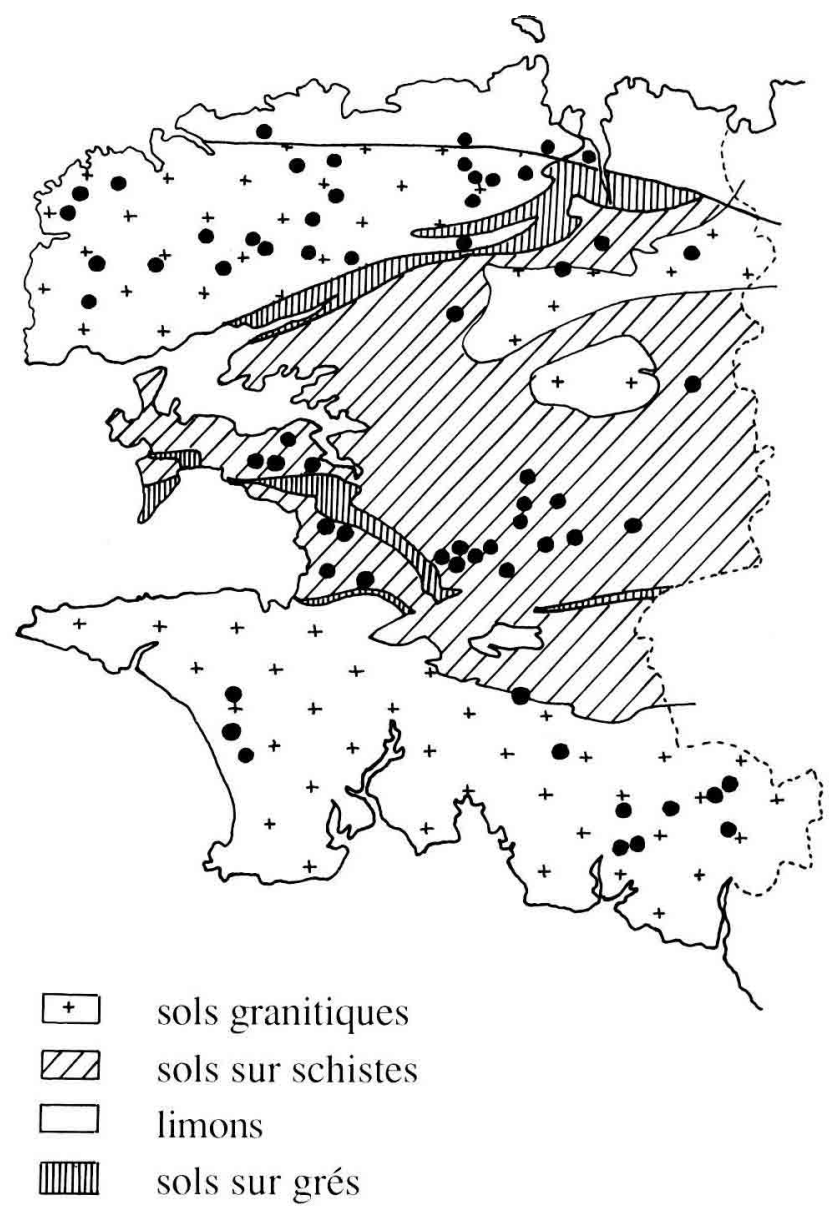

Fig 1. Carte pédologique du Finistère et situation des 64 exploitations agricoles suivies. 
Tableau I. Évolution de 1973 à 1988, des productions animales présentes sur les 64 exploitations enquêtées.

\begin{tabular}{lrrr} 
Système de production & 1973 & 1982 & 1989 \\
\hline & & & \\
\hline Porcs seuls & 19 & 29 & 27 \\
Porcs + laitières & 17 & 11 & 7 \\
Porcs + taurillons & 11 & 9 & 8 \\
Porcs + laitières + taurillons & 3 & 2 & 4 \\
Laitières & 2 & 1 & 1 \\
Laitières + taurillons & 1 & 2 & 2 \\
Taurillons ou veaux & 4 & 4 & 5 \\
Volailles seules & 2 & 2 & 2 \\
Volailles + divers & 5 & 4 & 4 \\
& & & \\
Total & 64 & 64 & 60
\end{tabular}

\section{Cultures pratiquées et fertilisation}

Six grands types de rotations culturales sont pratiquées dans ces exploitations :

- monoculture de maïs;

- maïs - ray-grass d'Italie;

- maïs - ray-grass anglais (ou fétuque élevée);

- maïs - céréales;

- prairie permanente;

- cultures légumières (chou-fleur - artichaut).

En 1973, le quart des parcelles suivies ne recevaient pas d'engrais minéraux phosphatés ou potassiques. Cette proportion a augmenté au cours du temps, en raison des accroissements de teneurs relevés lors des campagnes de mesure. Les quantités de lisier épandues sont généralement comprises entre 40 et 80 $\mathrm{m}^{3 / h a / a n}$ (moyenne 70). Seuls 9 éleveurs épandent plus de $100 \mathrm{~m}^{3} / \mathrm{ha} / \mathrm{an}$ et 20 cèdent du lisier à leurs voisins.

\section{Méthode d'enquête}

\section{Analyses de terre}

Les services techniques de la Chambre d'agriculture du Finistère ont effectué dans chaque exploitation un prélèvement de terre sur 3 parcelles définies au départ en fonction des assolements pratiqués. Un tel choix limite les éventuelles insuffisances de représentativité attribuables au faible nombre d'échantillons prélevés. Au total, 186 parcelles sont ainsi suivies depuis plus de 15 ans.

Les prélèvements de sol pour analyse ont été effectués selon la technique classique: 15 carottes d'une profondeur égale à celle de l'horizon cultivé, sont prélevées au hasard dans les zones initialement définies.
Les prélèvements de terre ont été effectués par les conseillers agricoles de la Chambre d'agriculture et les analyses de terre par la station d'agronomie de Quimper.

\section{Fiches d'enquête}

Une fiche d'enquête est remplie par l'agriculteur lors de chaque campagne de prélèvement, de façon à préciser :

- l'évolution de son système de production (culture et élevage);

- les cultures (type et production) et la fertilisation (quantités de lisier, engrais et amendements épandus) pratiquées sur les 3 parcelles au cours des 3 années précédant l'enquête;

- les principaux termes des bilans minéraux, uniquement lors de l'enquête la plus récente, en 1988.

\section{Déterminations réalisées}

Toutes les analyses chimiques de sol ont été effectuées par la station d'agronomie de Quimper. Ont été déterminées les teneurs du sol en :

- acide phosphorique $\left(\mathrm{P}_{2} \mathrm{O}_{5}\right.$ citrique, méthode DyerDemolon);

- potasse échangeable (acétate d'ammonium);

- cuivre et zinc EDTA (EDTA - 0,01 M dissous dans l'acétate d'ammonium neutre et normal; $2 \mathrm{~h}$ d'agitation de $5 \mathrm{~g}$ de terre dans $50 \mathrm{ml}$ de réactif).

Soulignons que les échantillons de terre prélevés lors de chaque campagne ont été soigneusement conservés. Ils peuvent ainsi être réutilisés pour répondre aux autres questions que peuvent poser l'utilisation massive des lisiers (incidences sur l'évolution des taux de matière organique, du $\mathrm{pH}$, des bases échangeables ou d'autres composantes de la fertilité).

\section{RÉSULTATS}

Ils illustrent l'évolution de la composition chimique des sols, mesurée par 6 campagnes de prélèvements échelonnées sur 15 ans.

\section{Acide phosphorique}

La teneur moyenne en acide phosphorique des sols, passe de $440 \mathrm{ppm}$ en 1973, à 855 en 1988 , soit une augmentation de $100 \%$ en 15 ans (fig 2). Cette augmentation est en relation avec les quantités de lisier épandues : $300 \mathrm{mg} \mathrm{P}_{2} \mathrm{O}_{5} /$ $\mathrm{kg}$ de terre pour les sols recevant moins de 50 


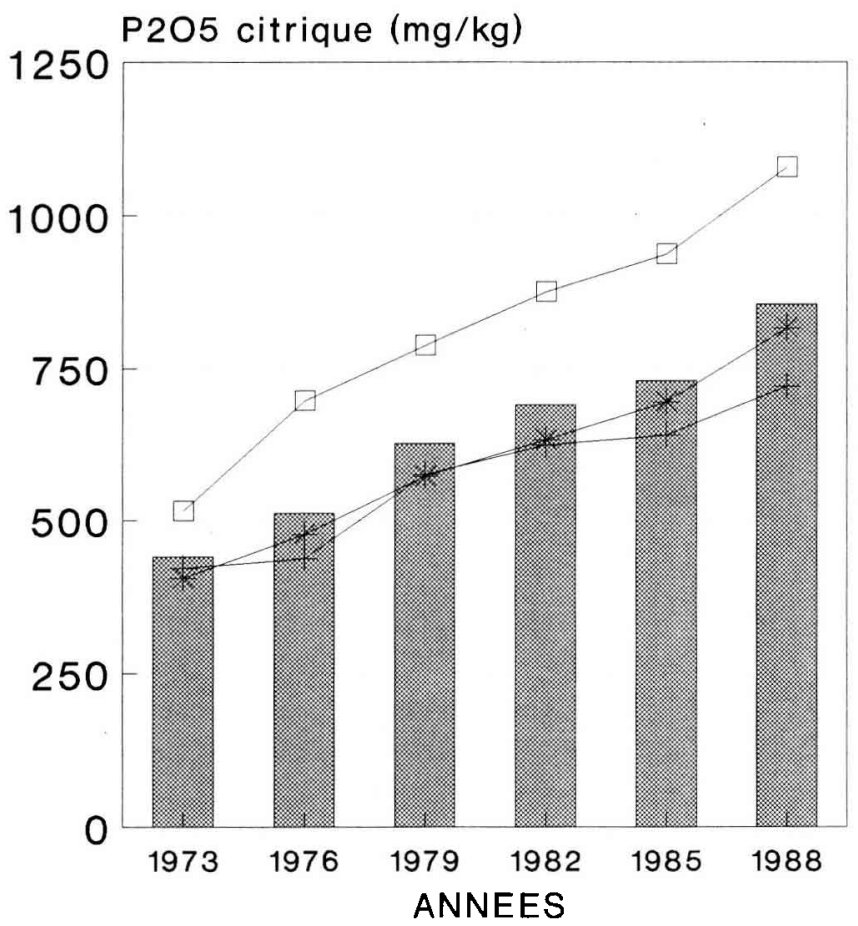

Fig 2. Évolution entre 1973 et 1988 , de la teneur en $\mathrm{P}_{2} \mathrm{O}_{5}$ critique $(\mathrm{mg} / \mathrm{kg}$ de terre) des sols d'exploitations d'élevages intensifs du Finistère en fonction de la dose annuelle de lisier épandue. Moyenne générale; - +- $<50 \mathrm{~m}^{3} \cdot \mathrm{ha}^{-1} \cdot \mathrm{an}^{-1}$; *-50-100 $\mathrm{m}^{3} \cdot \mathrm{ha}^{-1} \cdot \mathrm{an}^{-1} ;$ - $->100 \mathrm{~m}^{3} \cdot \mathrm{ha}^{-1} \cdot \mathrm{an}^{-1}$.

$\mathrm{m}^{3}$ de lisier/ha/an, 560 pour les sols recevant plus de $100 \mathrm{~m}^{3} / \mathrm{ha} / \mathrm{an}$. On constate dans ce dernier cas que la teneur moyenne des sols, en 1988 , dépasse $1000 \mathrm{mg}$ de $\mathrm{P}_{2} \mathrm{O}_{5} / \mathrm{kg}$ de terre. Rappelons que, dans le contexte local, un sol contenant $250 \mathrm{mg}$ de $\mathrm{P}_{2} \mathrm{O}_{5} / \mathrm{kg}$ de terre est considéré comme bien pourvu.

Nous observons un enrichissement moyen du sol de $28 \mathrm{mg}$ de $\mathrm{P}_{2} \mathrm{O}_{5} / \mathrm{kg}$ de terre/an, avec des écarts allant de 20 à $38 \mathrm{mg} / \mathrm{kg}$ de terre/an selon l'importance des apports de lisier.

Cet enrichissement peut être comparé à l'enrichissement théorique attendu : sachant que, dans les sols finistériens, la méthode Dyer permet d'extraire $66 \%$ de l'acide phosphorique apporté par le lisier (Coppenet et Golven, 1984), l'accroissement moyen observé au cours de l'enquête correspond à un apport moyen de $126 \mathrm{~kg}$ $\mathrm{P}_{2} \mathrm{O}_{5}$ ha $^{-1} \cdot \mathrm{an}^{-1}$.

Une telle comparaison a pu être réalisée sur un sous-échantillon de 23 exploitations spécialisées en production porcine, à partir des renseignements fournis par les agriculteurs lors de l'enquête de 1988. Pour ces exploitations, l'enri- chissement moyen annuel observé est de $31 \mathrm{mg} /$ $\mathrm{kg}$ de terre, ce qui correspond à un apport potentiel de $141 \mathrm{~kg} / \mathrm{ha} / \mathrm{an}$. Selon les indications fournies, les apports moyens (tant par les engrais minéraux que par le lisier) sont de l'ordre de $210 \mathrm{~kg}$ $\mathrm{P}_{2} \mathrm{O}_{5}$ ha $^{-1}$.an-1, auxquels il convient de retrancher environ $50 \mathrm{~kg} \mathrm{P}_{2} \mathrm{O}_{5}$ ha $^{-1}$. $\mathrm{an}^{-1}$ d'exportations, soit un reliquat annuel de $160 \mathrm{~kg}$. En dépit des difficultés à mettre en relation les apports et l'enrichissement correspondant du col (Coppenet et Golven, 1984; Destain, 1981), on note donc une certaine convergence entre l'évolution observée des teneurs et l'évolution prévisible par un calcul de bilan.

Dans l'état actuel des connaissances, cet enrichissement des sols en $\mathrm{P}_{2} \mathrm{O}_{5}$ n'est pas préoccupant pour la plante. En effet, en raison de l'antagonisme phosphore-zinc, on pourrait craindre l'apparition de carences zinciques sur maïs, mais les lisiers apportant également des quantités non négligeables de zinc, le risque d'apparition de telles carences est à écarter. Par ailleurs, les ions $\mathrm{PO}_{4}^{---}$, dont une partie reste soluble, peuvent entraîner une augmentation de la teneur en sels solubles et provoquer des brûlures aux racines. Des accidents de phytotoxicité provoqués par l'augmentation de la salinité résultant de l'accumulation de phosphates sont peu probables, des expérimentations en pots (apports équivalents à 1 à $60 \mathrm{t}$ de $\mathrm{P}_{2} \mathrm{O}_{5}$ ) n'ayant pas montré d'effet négatif sur des cultures tests à l'exception du pois (au-delà de 40 t $\mathrm{P}_{2} \mathrm{O}_{5} / \mathrm{ha}$ ).

En revanche, cet enrichissement peut être préoccupant pour l'environnement, le phosphore pouvant être entraîné vers les eaux superficielles, par érosion hydrique.

\section{Potassium}

La teneur moyenne des sols en potasse échangeable est passée en 15 ans de 306 à 408 ppm, soit une augmentation de $100 \mathrm{mg}$ (fig 3). Dans le contexte local, rappelons qu'un sol contenant $250 \mathrm{mg}$ de $\mathrm{K}_{2} \mathrm{O} / \mathrm{kg}$ de terre est considéré comme bien pourvu. L'accroissement de teneur est régulier pour les sols initialement les moins bien pourvus $\left(200 \mathrm{mg}\right.$ de $\mathrm{K}_{2} \mathrm{O} / \mathrm{kg}$ de terre), il est faible pour les sols initialement riches (400 mg de $\mathrm{K}_{2} \mathrm{O} / \mathrm{kg}$ de terre).

On assiste ainsi à un plafonnement des teneurs en potasse du sol vers $400 \mathrm{mg} \mathrm{K}_{2} \mathrm{O} / \mathrm{kg}$. Cette différence de comportement par rapport à $\mathrm{P}_{2} \mathrm{O}_{5}$ peut s'expliquer principalement par : 
$\mathrm{K} 2 \mathrm{O}$ échangeable $(\mathrm{mg} / \mathrm{kg})$

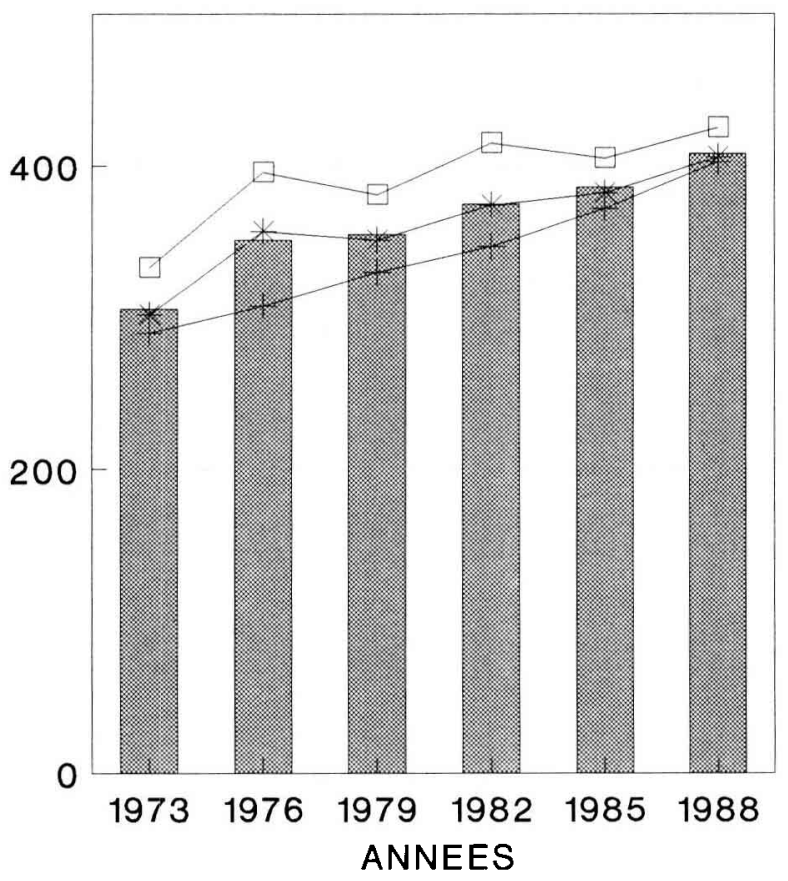

Fig 3. Évolution entre 1973 et 1988 , de la teneur en $\mathrm{K}_{2} \mathrm{O}$ échangeable ( $\mathrm{mg} / \mathrm{kg}$ de terre) des sols d'exploitations d'élevages intensifs du Finistère en fonction de la dose annuelle de lisier épandue. Moyenne générale; $-+-<50 \mathrm{~m}^{3}$ $\mathrm{ha}^{-1} \cdot \mathrm{an}^{-1}$; - ${ }^{*}-50-100 \mathrm{~m}^{3} \cdot \mathrm{ha}^{-1} \cdot \mathrm{an}^{-1} ;$ - - > $100 \mathrm{~m}^{3} \cdot \mathrm{ha}^{-1}$. $a^{-1}$.

- le lessivage du potassium non fixé par le sol (Simon et Le Corre, 1989);

- des exportations plus importantes de $\mathrm{K}_{2} \mathrm{O}$ par les plantes (consommation de luxe);

- la «rétrogradation" sous des formes peu solubles dans l'acétate d'ammonium d'une partie du potassium apporté.

L'accroissement moyen observé est de $6,8 \mathrm{mg}$ $\mathrm{K}_{2} \mathrm{O} / \mathrm{kg}$ de terre/an. Sur le même souséchantillon de 23 élevages de porcs charcutiers, un calcul comparable à celui effectué pour $\mathrm{P}_{2} \mathrm{O}_{5}$, conduit à un apport théorique de $20 \mathrm{~kg} \mathrm{~K} \mathrm{~K}_{2} \mathrm{O}$. $\mathrm{ha}^{-1} \cdot \mathrm{an}^{-1}$. Selon les indications fournies par les agriculteurs, les apports sont voisins de $150 \mathrm{~kg}$ $\mathrm{K}_{2} \mathrm{O} \cdot \mathrm{ha}^{-1}$.an-1. Si l'on retranche les exportations par les cultures $\left(30 \mathrm{~kg} \mathrm{~K} \mathrm{~K}_{2}\right.$.ha ${ }^{-1}$. an ${ }^{-1}$ pour le grain), et les pertes potentielles par lessivage (30 à $60 \mathrm{~kg} \mathrm{~K}_{2} \mathrm{O} \cdot \mathrm{ha}^{-1}$.an-1), le gain net calculé par parcelle est nettement supérieur à l'enrichissement effectivement observé. Cette diversité pourrait s'expliquer par une sous-estimation possible du lessivage ou de la rétrogradation en sols riches (Simon et Le Corre, 1989).

\section{Cuivre}

Le cuivre apporté dans les aliments destinés aux porcs en tant que facteur de croissance, n'est que très peu retenu par l'animal. On le retrouve donc dans ses déjections (Priem et Maton, 1980; Unwin, 1981; Coppenet, 1981; Mac Grath, 1981).

En 15 ans, la teneur moyenne en cuivre des parcelles suivies est passée de $3,82 \mathrm{ppm}$ à 7,10 $\mathrm{ppm}$, soit une augmentation de 0,22 ppm/an (fig 4). Cette augmentation est d'autant plus importante que la dose de lisier épandue est forte : 2,65 ppm en 15 ans pour moins de $50 \mathrm{~m}^{3}$ $\mathrm{ha}^{-1} \cdot \mathrm{an}^{-1}, 3,33 \mathrm{ppm}$ de 50 à $100 \mathrm{~m}^{3} \cdot \mathrm{ha}^{-1} \cdot \mathrm{an}^{-1}$, et $4,27 \mathrm{ppm}$ pour plus de $100 \mathrm{~m}^{3} \cdot \mathrm{ha}^{-1}$.an-1.

On rappelera que dans ce type de sols, la carence en cuivre est probable pour une teneur inférieure à $1 \mathrm{ppm}$, et la toxicité est à craindre pour des teneurs supérieures à 120 ppm.

Bien que nous ne soyons pas en mesure de préciser exactement la proportion de cuivre incorporée au sol qui peut être ensuite extraite par le réactif utilisé, on peut tenter de mettre en parallèle l'enrichissement du sol et les apports correspondants en s'appuyant sur les observations de Coppenet (1981) : si l'on fait l'hypothèse qu'une attaque à l'acide nitroperchlorique extrait

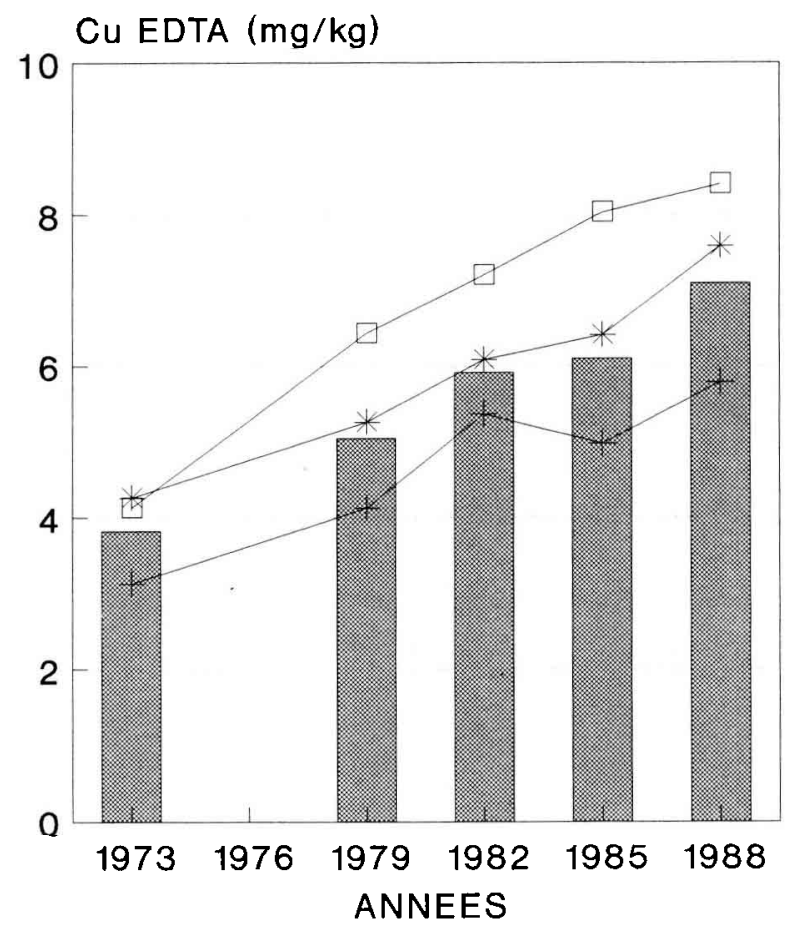

Fig 4. Évolution entre 1973 et 1988 , de la teneur en Cu EDTA ( $\mathrm{mg} / \mathrm{kg}$ de terre) des sols d'exploitations d'élevages intensifs du Finistère en fonction de la dose annuelle de lisier épandue. Moyenne générale; - $-<50 \mathrm{~m}^{3} \cdot \mathrm{ha}^{-1} \cdot \mathrm{an}^{-1}$; — 50-100 m $\mathrm{m}^{3} \cdot \mathrm{ha}^{-1} \cdot \mathrm{an}^{-1} ;-1$ - $>100 \mathrm{~m}^{3} \cdot \mathrm{ha}^{-1} \cdot \mathrm{an}^{-1}$. 
la totalité du cuivre apporté, il apparaît que la méthode EDTA utilisée en extrait environ $60 \%$.

Pour le sous-échantillon de 23 exploitations porcines, les analyses de terre successives mettent en évidence un enrichissement effectif de $0,24 \mathrm{ppm} \mathrm{Cu}$ extractible par EDTA par an. Le calcul effectué à partir de la fiche d'enquête conduit à des apports théoriques voisins de $1,17 \mathrm{~kg} \mathrm{Cu} / \mathrm{ha} / \mathrm{an}$, auxquels il convient de retrancher les exportations par les cultures $(50 \mathrm{~g} \mathrm{Cu} /$ ha/an), soit un enrichissement calculé de 0,37 ppm Cu par an (1,12 kg Cu dans 3000 tonnes de terre). Si l'on retient l'hypothèse que la méthode utilisée n'extrait que $60 \%$ du cuivre apporté, l'enrichissement observé devrait être de 0,2 ppm, ce qui est en bon accord avec l'enrichissement constaté.

\section{Zinc}

Le zinc est incorporé aux aliments des porcs pour éviter une maladie de la peau, la parakératose. Le taux d'incorporation de zinc aux aliments est voisin de $125-150 \mathrm{mg} \mathrm{Zn/kg}$ d'aliment. Le zinc étant peu retenu par l'animal, les lisiers en sont toujours très riches ( 15 à $20 \mathrm{~g} / \mathrm{m}^{3}$ ).

En 15 ans, les teneurs moyennes des sols sont passées de $3,19 \mathrm{ppm}$ à $8,76 \mathrm{ppm}$, soit une augmentation annuelle de $0,37 \mathrm{ppm}$ (fig 5). Cette dernière est, comme pour le cuivre, d'autant plus élevée que la quantité de lisier épandue est importante : 0,23 ppm pour moins de 50 $\mathrm{m}^{3}$ de lisier/ha/an, $0,33 \mathrm{ppm}$ de 50 à $100 \mathrm{~m}^{3}$, et $0,58 \mathrm{ppm}$ pour plus de $100 \mathrm{~m}^{3}$. Les carences en zinc peuvent être rencontrées sur plantes sensibles (maïs) lorsque le sol a une teneur inférieure à 1,5 ppm de $\mathrm{Zn}$. La phytotoxicité apparaît quand la teneur du sol dépasse $120 \mathrm{ppm}$.

Comme pour les éléments précédents, on peut faire la comparaison entre l'enrichissement ob-servé dans le sol et les apports, sur le même sous-échantillon de 23 exploitations porcines. Les analyses de terre mettent en évidence un enrichissement moyen de $0,41 \mathrm{ppm} / \mathrm{an}$. Le calcul du bilan du zinc sur ces exploitations conduit à un apport moyen de $2,94 \mathrm{~kg} \mathrm{Zn/ha/an} \mathrm{d'où} \mathrm{l'on}$ doit retrancher les exportations (environ $200 \mathrm{~g}$ $\mathrm{Zn} / \mathrm{ha} / \mathrm{an}$ ), soit un enrichissement potentiel de $0,91 \mathrm{ppm}$ par an. Si l'on retient l'hypothèse que la méthode utilisée n'extrait que $40 \%$ du zinc apporté (Coppenet, 1981), l'enrichissement observé devrait être de $0,37 \mathrm{ppm}$, ce qui est voisin de l'augmentation de teneur constatée.

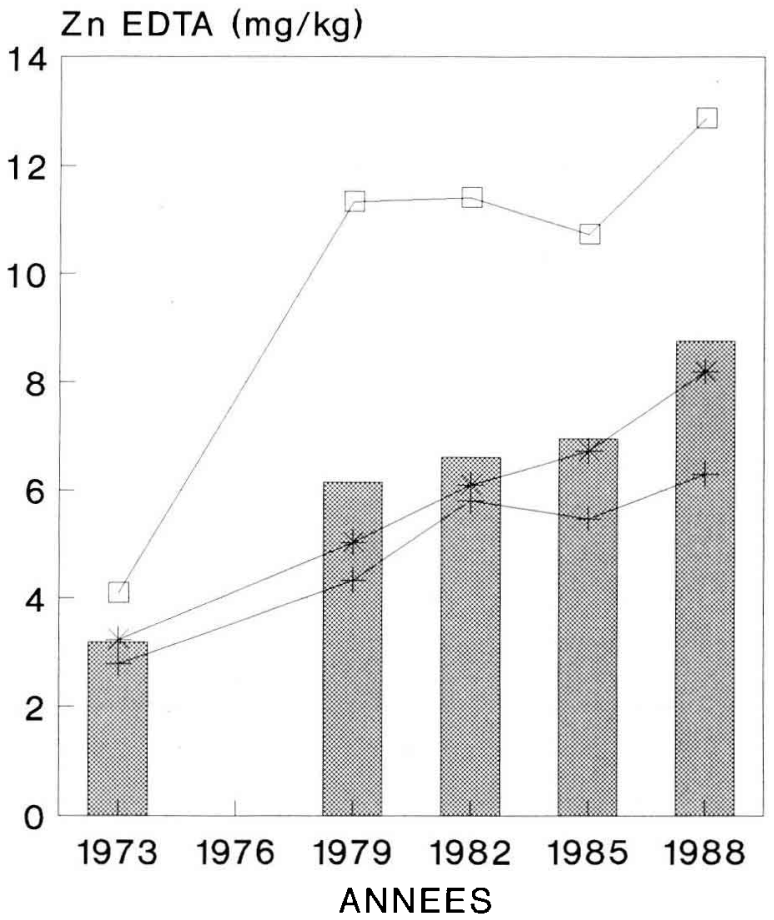

Fig 5. Évolution entre 1973 et 1988 de la teneur en Zn EDTA ( $\mathrm{mg} / \mathrm{kg}$ de terre) des sols d'exploitations d'élevages intensifs du Finistère en fonction de la dose annuelle de lisier épandue. Moyenne générale; $-+<50 \mathrm{~m}^{3} / \mathrm{ha} / \mathrm{an} ;-$ *⿻丷木- -50 $100 \mathrm{~m}^{3} / \mathrm{ha} / \mathrm{an} ;$ - $\longrightarrow 100 \mathrm{~m}^{3} / \mathrm{ha} / \mathrm{an}$.

À très long terme, une phytotoxicité du zinc est donc à craindre (Coppenet, 1975 et 1981; Meeus-Verdinne et al, 1980; Lexmond et de Haan, 1980). Celle-ci peut s'ajouter à celle du cuivre (Coppenet, 1981; Beckett, 1981). Pour les sols de Bretagne occidentale dont le $\mathrm{pH}$ eau est voisin de 6, la phytotoxicité due au cumul de $\mathrm{Cu}$ et $\mathrm{Zn}$ s'observe dès que Cu EDTA + Zn EDTA est supérieur à $120 \mathrm{ppm}$. Ces phytotoxicités irréversibles pourraient apparaître à échéance d'un siècle si les tendances actuelles perdurent. Une diminution de l'incorporation de ces 2 éléments aux aliments, déjà importante pour le cuivre, est de ce fait vivement préconisée par les agronomes.

\section{DISCUSSION ET CONCLUSION}

Si l'analyse de 3 parcelles ne permet pas de caractériser précisément les modifications chimiques des sols d'une exploitation, l'enquête effectuée dans le Finistère montre néanmoins qu'en terme de tendances, les prévisions théoriques avancées dès 1974 se confirment. 
Soumis aux apports répétés de fortes quantités de lisier (essentiellement de porc dans cette enquête), les sols s'enrichissent fortement en acide phosphorique, en cuivre et zinc, et plus modérément en potasse.

L'enrichissement en acide phosphorique assimilable est très important, comme ont pu le montrer divers auteurs (Furrer, 1981). Cette accumulation résulte d'une forte fixation du phosphore sur le complexe argilo-humique du sol. Le phosphore s'accumule dans les horizons superficiels (Destain, 1981) et ne migrerait que très peu en profondeur. Les très faibles quantités de phosphore mesurées dans les eaux de drainage en témoignent (Simon et Le Corre, 1989; Johnston, 1981; Unwin, 1981). La forte augmentation de la teneur en $\mathrm{P}_{2} \mathrm{O}_{5}$ du sol est observée même dans les parcelles où l'on n'emploie pas de phosphates minéraux. Cette observation doit donc inciter à suspendre l'application de ce type d'engrais dans les parcelles recevant des apports réguliers de lisier.

Les augmentations de teneur du sol sont particulièrement préoccupantes pour le cuivre et le zinc. II serait intéressant que les zootechniciens diminuent encore la complémentation des aliments en ces deux métaux. Si les apports de zinc ne peuvent être diminués, il conviendra de surveiller son accumulation dans les couches arables par des analyses régulières.

Les éléments fertilisants contenus dans les déjections animales sont désormais couramment pris en compte pour l'établissement des plans de fumure. Cette évolution a déjà permis d'importantes économies sur les achats d'engrais phosphatés dans ces exploitations d'élevage. II conviendrait qu'une évolution semblable se dessine également pour l'azote, pour lequel un calcul analogue de bilan met également en évidence de très importants excédents pour ce type d'exploitation (Simon et Le Corre, 1992), sans que l'on note pour autant une diminution des achats d'engrais minéraux dans les statistiques départementales annuelles.

\section{RÉFÉRENCES}

Beckett P (1981) Copper in sludge: are the toxic effects of copper and other heavy metals additive? In: Copper in animals wastes and sewage sludge $D$ Reidel Publishing Company, London, 204-222

Coppenet M (1972) Le bilan des éléments fertilisants dans les exploitations d'élevage. À la pointe de l'élevage (revue de l'EDE du Finistère), 34, 2-5
Coppenet M (1974) L'épandage du lisier de porcherie. Ses conséquences agronomiques. Ann Agron 25 (2-3), 403-423

Coppenet M (1975) Bilan des éléments fertilisants sur les exploitations d'élevage. Fourrages 62, 119-132

Coppenet M (1981) Copper accumulation in Brittany soils through enriched pig slurry; phytotoxic risks. In: Copper in animals wastes and sewage sludge. D Reidel Publishing Company, London, 154-161

Coppenet M (1985) Bilan des éléments fertilisants dans les exploitations d'élevage : application à une fertilisation raisonnée. Tech Agric 1315, 12 p

Coppenet M, Golven J (1984) Enquête «lisier-solplante" : bilan de 10 années de suivi dans une soixantaine d'exploitations intensives du Finistère. Brochure éditée par la Chambre d'agriculture du Finistère et la station d'agronomie de Quimper, $33 \mathrm{p}$ + annexes

Destain JP (1981) The effect of very intensive dressings of livestock effluent on phosphorus in soil. In: Phosphorus in sewage sludge and animal wastes slurries. D Reidel Publishing Company, London, 371-381

Furrer OJ (1981) Accumulation and leaching of phosphorus as influenced by sludge application. In: Phosphorus in sewage sludge and animal wastes slurries. D Reidel Publishing Company, London, 235-240

Johnston AE (1981) Accumulation of phosphorus in a sandy loam soil from farmyard manure and sewage sludge. In: Phosphorus in sewage sludge and animal wastes slurries. $D$ Reidel Publishing Company, London, 273-290

Lexmond TM, de Haan FAM (1980) Toxicity of copper. In: Effluents from livestock (JKR Gasser, ed). Applied Science Publishers, Barking, 410-419

Mac Graff MC (1981) Application applying copper rich pig slurry to grassland; effects on plant and soil. In: Copper in animals wastes and sewage sludge. D Reidel Publishing Company, London, 144-153

Meeus-Verdinne K, Neirinckx G, Monseur X, de Borger $R$ (1980) Real or potentiel risk of pollution of soils, crops, surface and groundwater due to the landspreading of liquid manure. In: Effluents from livestock (JKR Gasser, ed). Applied Science Publishers, Barking, 399-409

Priem R, A Maton (1980) The influence of the content of trace elements in the feed on the composition of liquid mature of pigs. In: Effluents from livestock (JKR Gasser, ed). Applied Science Publishers, Barking, 9-22

Simon JC, Le Corre L (1989) Lessivage d'éléments minéraux autres que l'azote en monoculture de maïs, en sol granitique du Finistère. Fourrages $118,127-148$

Simon JC, Le Corre L (1992) Le bilan de l'azote à l'échelle de l'exploitation agricole : méthodologie; exemples de résultats. Fourrages 129, 79-94

Unwin RJ (1981) Phosphorus accumulation and mobility from large applications of slurry. In: Phosphorus in sewage sludge and animal wastes slurries. D Reidel Publishing Company, London, 333-346 